\title{
Calidad de la educación superior tecnológica en Iberoamérica
}

\author{
Félix Pizango Taricuarima \\ felpita_1957@hotmail.com \\ https://orcid.org/0000-0001-9806-1642 \\ Oscar Alejandro Tuesta Hidalgo \\ oscartuestahidalgo@gmail.com \\ ORCID: 0000-0003-2231-9365 \\ Juan Carlos Tuesta Hidalgo \\ Jcth3@yahoo.es \\ https://orcid.org/0000-0002-2959-1129 \\ Roberto Alejandro Pacheco Robles \\ tatorpr@hotmail.com \\ https://orcid.org/. 0000-0003-3406-1322. \\ Dr. Hipólito Percy Barbaran Mozo \\ pbarmozito@hotmail.com \\ https://orcid.org/0000-002-9316-202X
}

\section{RESUMEN}

El presente artículo tiene como objetivo analizar la calidad de la educación superior tecnológica en Iberoamérica, mediante una revisión sistemática, dado que el procedimiento implica un análisis integrativo y retrospectivo de las investigaciones realizadas y publicadas entre los años 2018 - 2020. En este sentido, se muestran algunas relaciones materializadas en nociones que exponen la mirada sobre las transformaciones culturales y educativas generadas por los desarrollos de la tecnología. El artículo se organiza en tres secciones que presentan los modos conceptuales sobre la relación educación y tecnología: el análisis pedagógico al efecto de los medios en educación; la búsqueda de la inclusión con el uso de la tecnología, la educación virtual, la subjetividad y experiencia de interacción en entornos tecnológicos. Entre las principales conclusiones, se destaca que, la calidad de la educación tecnológica como parte de un sistema educativo, tanto en el Perú, como en otros países iberoamericanos presenta baja valoración en el sector productivo y poco prestigio social, debido a su precariedad en el equipamiento y 
servicio educativo, debido a la escasa articulación con las demandas laborales y las necesidades del desarrollo local, regional y nacional; sumándose otros indicadores educativos o condiciones mínimas de calidad que deben tener las instituciones públicas o privadas que brindan este servicio; propiciando la cabida de los procesos de mejora continua de la calidad para promover el valor de la optimización, el licenciamiento y la acreditación como estrategia para alcanzar la calidad.

Palabras clave: calidad; educación tecnológica; desarrollo tecnológico. 


\title{
Quality of technological higher education in Iberoamerica
}

\begin{abstract}
The objective of this article is to analyze the quality of technological higher education in Latin America, through a systematic review, since the procedure involves an integrative and retrospective analysis of the research carried out and published between the years 2018 - 2020. In this sense, it is They show some relationships materialized in notions that expose the view on the cultural and educational transformations generated by the developments of technology. The article is organized into three sections that present the conceptual modes of the relationship between education and technology: the pedagogical analysis of the effect of the media in education; the search for inclusion with the use of technology, virtual education, subjectivity and experience of interaction in technological environments. Among the main conclusions, it stands out that the quality of technological education as part of an educational system, both in Peru and in other Ibero-American countries, presents low value in the productive sector and little social prestige, due to its precariousness in the educational equipment and service, due to the scarce articulation with labor demands and the needs of local, regional and national development; adding other educational indicators or minimum quality conditions that the public or private institutions that provide this service must have; fostering the space of continuous quality improvement processes to promote the value of optimization, licensing and accreditation as a strategy to achieve quality.
\end{abstract}

Keywords: quality; technological education; technological development.

Artículo recibido: 05 octubre. 2021 Aceptado para publicación: 02 noviembre 2021 Correspondencia: felpita_1957@hotmail.com Conflictos de Interés: Ninguna que declarar 


\section{INTRODUCCIÓN}

Evidentemente los logros alcanzados en la ciencia y la tecnología han dado un giro con respecto a la visión en el mundo en estos últimos años. En cambio, una gran parte de la sociedad constantemente se desvincula de estas variaciones, ocasionando con ello una nueva forma de exclusión, lo cual limita a las personas de las herramientas para un mejor desenvolvimiento en este mundo tecnológico. Esta situación permite inferir que la educación tecnológica se centra en una serie de temas o contenidos que deben ser abordados en el sistema educativo orientados a comprender el uso de las diversas tecnologías en un contexto sociocultural; es decir, implica capacitación, instrucción y desarrollo de habilidades sobre un determinado equipo tecnológico; a fin de entender, primero para qué sirve, cómo funciona, cuál es el propósito para el que fue creado, qué necesita el usuario para su uso adecuado y evitar daños, etc.

En efecto, la educación tecnológica es una de las innovaciones en las reformas educativas, que busca desarrollar un modelo de pensamiento en estos tiempos de pandemia, ya que relaciona el "pensar" con el "hacer", desde el nivel inicial, primaria, secundaria y superior; de esta manera, lograr capacidades prácticas que permitan resolver problemas complejos, con efectos concretos en la realidad.

A modo de remembranza, la educación tecnológica se ha instaurado en España, Gran Bretaña y en los Estados Unidos, como un área que va dirigido a todos los estudiantes y no solo para aquellos que van a seguir carreras técnicas. En América Latina Argentina y Chile fueron los pioneros en incluir la enseñanza de la tecnología, seguidamente de Bolivia y Colombia. Implementar la educación tecnológica en cada uno de estos escenarios, se basó fundamentalmente en las peculiaridades propias de cada situación, contexto, experiencia y trayectoria pedagógica. El fin que se persigue es brindar una formación integral de calidad en la educación superior tecnológica a los estudiantes. Por ningún motivo se busca la formación para una ocupación específica, sino desarrollar una cultura tecnológica y propiciar a los estudiantes las herramientas que les permita comprender al mundo superficial que les rodea.

Por otro lado, las Nuevas Tecnologías de la Información y Comunicación (NTICs), no son solamente una necesidad en la sociedad actual, sino que lideran la transición hacia la cuarta revolución industrial (World Economic Forum, 2016), basada en la conectividad; por ello tener competencias en su uso es un requisito indispensable para el acceso al 
mundo laboral (Organization of Economic Co-operation and Development, 2016). En este marco, el presente trabajo aborda la importancia de tener presente que los acelerados cambios imprimidos en las tendencias de mercado no siempre se traducen con la misma agilidad y eficiencia requeridas en la educación superior, por lo que nuestros estudiantes se apropien del uso adecuado de las nuevas tecnologías y su aprendizaje.

El desarrollo tecnológico en diferentes aspectos, representan una herramienta de gran impacto para el desarrollo económico de los países, y es necesaria su integración en la educación puesto que son indispensables en la globalización y facilitan la participación ciudadana (Benavides \& Pedró, 2007; Kozma, 2008). El trabajo de López (2011) resaltó aspectos relevantes acerca de las recomendaciones desde organismos internacionales para el diseño de las políticas públicas en materia de educación y tecnologías, y en qué aspectos situaron el foco de importancia para desarrollar sus propuestas.

En esa línea de investigación, Melo (2017) plantea que la Unesco y el Banco Mundial (BM) situaron el eje en torno a la importancia de la figura del docente como responsable desde el aula para la inclusión de las NTICs en el medio educativo tradicional, apuntando a que debería ser prioritario que los gobiernos fortalezcan la formación de docentes junto con habilitar equipos en las instituciones educativas. Por otro lado, instituciones como la Organization of Economic Cooperation and Development (OECD), otorgan un papel de mayor importancia al estudiante, atendiendo al creciente uso intensivo de tecnologías que realiza fuera del aula y subrayando la necesidad de establecer vínculos en el uso no educativo con los aspectos de formación (OECD, 2016). En una visión complementaria a las anteriores, el Banco Interamericano de Desarrollo (BID) pone el énfasis en los aprendizajes como objetivo central de la integración de las NTICs en la educación aportando una visión integrada (Claro, 2010).

$\mathrm{Al}$ respecto de dicha diversidad de criterios, cabe la reflexión previa de qué se espera al introducir las NTICs en la educación; (Benavides y Pedro, 2007) situaron el énfasis de las políticas educativas en cuatro necesidades a las cuales contribuiría la implementación de las NTICs en el sistema educativo: (a) impulsar desarrollo económico, (b) promover equidad y justicia social, generando igualdad de oportunidades; (c) condicionar un cambio de modelo pedagógico y (d) apuntar a la calidad de aprendizaje.

Señalan los autores, que la respuesta inicial ante este panorama de directrices y necesidades de actuación por parte de los gobiernos se resolvió mayoritariamente 
mediante la inversión en dotación de equipos informáticos, facilitando el acceso a internet y financiando el desarrollo de contenidos para que ello redundara en el establecimiento de condiciones favorables para el uso de las NTICs en las aulas.

La prioridad de obtener resultados visibles en la agenda política ha impulsado la inversión en compra de equipamientos, presuponiendo que la presencia en el aula de herramientas conlleva el cambio de un enfoque educativo tradicional a uno mediatizado por las NTICs, recibiendo críticas ante la necesidad de trabajar para una apropiación social del medio para hacer efectivo su uso (Martí-Noguera \& Martí-Vilar, 2015). Como señala el trabajo de Lugo (2010), en atención a las recomendaciones de la Unesco y Banco Mundial sobre prestar atención a los docentes y el BID en los procesos de aprendizaje, sería preciso definir nuevos roles que permitan a los docentes estar cualificados para no tan solo impartir docencia virtual bajo el mismo modelo pedagógico, sino gestionar una nueva metodología que empodere la autonomía del estudiante como actor en su formación y no tan solo sea receptor pasivo. Sin apuntar como meta en los docentes la comprensión del uso de las tecnologías, resulta en vano llenar de recursos materiales tecnológicos las aulas (Kozma, 2008). Por ello, las estrategias de la agenda política deben enfatizar, como señala la Unesco, un cambio pedagógico que permita dotar a los docentes en las competencias pedagógicas que exige el modelo y no reducirse solamente a competencias tecnológicas. En base a ello, la evaluación del impacto de políticas TICs en la Educación no se debe limitar a medir los resultados de progreso a través de índices de consumo de dispositivos y el grado de avance de las interconexiones de internet, por lo que aseveran Andrade y Campo Redondo (2012) que las políticas impulsadas en los planes de desarrollo en la educación deben centrarse en analizar su implementación social.

Según la OECD (2006), los docentes son responsables en desarrollar en sus estudiantes competencias en el uso de tecnologías para incrementar la productividad, ello debe ser analizado desde un enfoque pedagógico y no solamente desde un impacto económico. La implementación y éxito en la apropiación de las NTICs en la educación requieren de la comprensión del marco teórico y metodológico adecuado para el aprendizaje en un nuevo contexto, así como un trabajo en la definición de perfiles profesionales docentes que deben implementar tecnologías cambiantes en el aula adaptando contenidos a nuevos medios y definiendo procesos de interacción con estudiantes. 
En este sentido, Martí-Noguera y Martí-Vilar (2015) hacen referencia a que la comprensión de la gestión de las NTICs en la Educación Superior requiere atender a dos aspectos, por una parte (a) directrices políticas que generen un marco metodológico de implementación con atención a las recomendaciones de expertos internacionales; y por otra parte (b) el conocer pautas de apropiación social de las NTICs en la educación como herramientas indispensables para relacionarse en un entorno global de conocimiento accesible desde internet. Tras haber comentado anteriormente los apuntes de organismos internacionales, en este apartado se analizan los estudios sobre el proceso de implementación en el aula.

Acorde con Gallardo-Echenique et al. (2015) en la apropiación de las NTICs en el contexto educativo hay que tener presentes las diferencias de pretender desarrollar la denominada competencia digital para saber usar las herramientas tecnológicas, con la orientación de la política hacia la alfabetización digital que requiere impulsar un paradigma adaptado a las dinámicas futuras y presentes; esta situación implica la formación digital continua a los profesores, así como investigación que permita evaluar resultados no limitándose solamente a acciones para capacitar sino estrategias para entender una herramienta en constante cambio. En concordancia, Rochefort y Richmond (2011) se refieren a la exigencia social de que las universidades se responsabilicen no solamente de crear un entorno virtual que prepare a estudiantes para entrar en el mercado de trabajo actual, sino que garantice un acceso continuo a la actualización de conocimientos, así como las competencias de interrelacionar conceptos y aplicarlos a un nuevo esquema de gestión de conocimiento. Sin embargo, remarcan los autores que la realidad de la apropiación de las tecnologías en las universidades dista de estar actualizada respecto de dichas tendencias, señalando que si bien "es posible que algunos profesores conozcan las tecnologías disponibles e incluso lleguen a usarlas, en general, los administradores y el equipo docente desconocen lo poderosas que pueden llegar a ser estas herramientas para la docencia” (Rochefort y Richmond, 2011, p. 203)

Analizando el proceso de interrelación entre la formación y las tecnologías, para Codina (2009) y Salavisa y Fontes, (2012) la apropiación de las NTICs en la educación necesita de una serie de procesos de adaptación en el centro educativo a la construcción de un espacio digital en el cual se facilite y acomoden dinámicas de uso de tecnologías ya presentes en gran parte de la sociedad conectada a la red, donde la ciudadanía participa 
en redes sociales lúdicas (Facebook, twitter) o laborales (LinkedIn), considerando necesario relacionar las TICs informales y comerciales con las educativas en base a atender la amalgama de intereses de la sociedad de innovación y conocimiento en el S. XXI. Esta apropiación tecnológica informal ya fue señalada por Kozma (2008), al indicar que la agenda política de implementación de las NTICs en la Educación Superior debía tener presente que: El sistema educativo peruano está organizado en dos etapas: Educación básica y Educación superior. La primera etapa es obligatoria y gratuita cuando la oferta el Estado, la segunda etapa está orientada a consolidar la formación integral de la persona, producir conocimiento, desarrollar la investigación e innovación y formar profesionales de alto nivel de especialización y perfeccionamiento en los campos del saber, arte, cultura, ciencia y tecnología, para cubrir la demanda de la sociedad y contribuir al desarrollo y sostenibilidad del país. La tecnología no es algo ajeno a la sociedad, existe una apropiación de las NTICs, con unos avances y evoluciones constantes que facilitan su uso en todas las capas poblacionales, al margen del sistema educativo sin que las políticas tengan margen de reacción y adaptación.

En el contexto del Perú, la educación superior tecnológica forma parte de la segunda etapa en el sistema educativo y está regida por la Ley No 29394, Ley de Institutos y Escuelas de Educación Superior; esta fue derogada por la Ley $\mathrm{N}^{\circ} 30512$, Ley de Institutos y Escuelas de Educación Superior y de la Carrera Pública de sus Docentes, y su reglamento aprobado mediante Decreto Legislativo $\mathrm{N}^{\circ} 010-2017-\mathrm{MINEDU}$ (Ministerio de Educación, 2017) ... según este marco normativo, se precisa que la educación tecnológica tiene como finalidad formar jóvenes que gestionen de manera sostenible los recursos naturales, culturales y paisajísticos, asimismo, formar personas calificadas en el manejo y conocimiento de los procesos tecnológicos de los diferentes sectores productivos, mejorar el pensamiento práctico, creativo y crítico, tomar decisiones en la solución de problemas, potenciar la capacidad de organización, la creatividad, la responsabilidad, liderazgo y valores para participar de manera eficiente y eficaz en el desarrollo de la economía nacional.

En esa perspectiva, los procesos de enseñanza-aprendizaje se relacionan con los procesos de producción, generándose una simbiosis. Por un lado, la formación profesional desarrolla las competencias requeridas por las empresas y el sector productivo; y, por otro lado, estas también reciben las innovaciones tecnológicas que en los institutos de 
educación superior tecnológica se desarrollan. Sin embargo, la actual calidad de la educación tecnológica en el Perú tiene una baja valoración en el sector productivo y poco prestigio social, debido a su precariedad en el equipamiento y servicio y la escasa articulación con las demandas laborales y las necesidades del desarrollo local, regional y nacional; sumándose otros indicadores educativos. En ese contexto, los procesos de mejora continua de la calidad educativa encuentran terreno fértil para promover el valor de la optimización, el licenciamiento y la acreditación como un instrumento de progreso económico y social.

\section{ESTARTEGIA METODOLÓGICA}

La presente investigación es una revisión sistemática, dado que el procedimiento implica el rastreo, organización, análisis y sistematización de un conjunto de artículos de revisión sobre el tema de la educación superior tecnológica, en el período comprendido entre los años 2018 - 2020. Las unidades de análisis fueron todos aquellos documentos sobre el tema, encontrados en las páginas web de reconocidas revistas indexadas.

Como criterios de búsqueda, se incluyeron los siguientes descriptores: "educación superior tecnológica", "educación superior no universitaria", "institutos tecnológicos", “educación superior técnico-productiva", y los institutos tecnológicos” y “optimización, licenciamiento y acreditación de institutos tecnológicos". Estos descriptores fueron combinados de diversas formas al momento de la exploración con el objetivo de ampliar los criterios de búsqueda.

Al realizar la búsqueda de los documentos, en cada una de las bases de datos, se preseleccionaron 20 artículos, de los cuales ocho seleccionamos, de acuerdo con los criterios de inclusión y exclusión. No se tomaron en consideración para el análisis aquellos artículos que no hacían alusión a los núcleos temáticos y/o aquellos que no se encontraban en revistas o instituciones reconocidas en la temática con el propósito de manifestar, de manera explícita, la sostenibilidad de la educación superior tecnológica en los institutos a través de la ejecución de proyectos productivos con la participación de los docentes. los estudiantes, el sector empresarial y el estado.

La primera fase de esta investigación correspondió a una búsqueda documental, encaminada a recolectar, comparar y analizar estudios sobre la educación superior no universitaria y educación tecnológica productiva. Para la selección de cada uno de los documentos o estudios que fueron revisados, se emplearon criterios como la relevancia y 
pertinencia al tema tratado, la valoración de la veracidad y confiabilidad científica de los resultados expresados sobre la base de corresponder a repositorios universitarios o revistas de investigación reconocidos; finalmente el último criterio fue la temporalidad, es decir, que no tengan una antigüedad mayor a cinco a años. 


\section{RESULTADOS Y DISCUCIÓN}

Tabla 1. Investigaciones sobre la sostenibilidad de la Educación Superior Tecnológica

\begin{tabular}{|c|c|c|c|c|c|c|c|}
\hline $\mathbf{N}^{\circ}$ & Año & Nombre del artículo & Revista & Autore & & Metodología & Resultados \\
\hline 1 & 2018 & $\begin{array}{lrr}\text { Revisión } & \text { de } & \text { la } \\
\text { educación } & \text { y } & \text { la } \\
\text { tecnología } & \text { desde } \\
\text { una } & \text { mirada } \\
\text { pedagógica } & \end{array}$ & $\begin{array}{l}\text { Revista Pedagogía y Saberes, } \\
\text { en el periodo 1990-2017 }\end{array}$ & Acevedo & & $\begin{array}{l}\text { Tipo básica, } \\
\text { nivel } \\
\text { explicativo. }\end{array}$ & $\begin{array}{l}\text { Se trata de un ejercicio exploratorio fundamentado en } \\
\text { el rastreo documental y categorial de artículos } \\
\text { publicados. El análisis propone que, para comprender } \\
\text { dicho campo, es necesario superar los límites de la } \\
\text { escuela y los roles asignados a ella tradicionalmente. }\end{array}$ \\
\hline 3 & 2017 & $\begin{array}{l}\text { Las Tic en la } \\
\text { educación superior, } \\
\text { innovaciones r y } \\
\text { retos. }\end{array}$ & $\begin{array}{l}\text { Revista Iberoamericana de la } \\
\text { Ciencias Sociales y } \\
\text { Humanísticas. Vol. 6, Núm. 12, } \\
\text { México }\end{array}$ & $\begin{array}{l}\text { García, } \\
\text { Reyes } \\
\text { Godínez }\end{array}$ & $\mathrm{y}$ & $\begin{array}{l}\text { Tipo básica, } \\
\text { diseño } \\
\text { descriptivo. }\end{array}$ & $\begin{array}{l}\text { Las TIC están ofreciendo a los estudiantes acceso a } \\
\text { fuentes de conocimiento ilimitados, a herramientas } \\
\text { multimedia que permiten ampliar estos conocimientos } \\
\text { de información. Sin duda, las TIC han transformado el } \\
\text { entorno de aprendizaje actual, pasando de uno } \\
\text { tradicional centrado en el docente a uno centrado en el } \\
\text { alumno, ya que el primero ha dejado de ser la principal } \\
\text { fuente de información y el principal emisor de } \\
\text { conocimiento para convertirse en un guía o conductor } \\
\text { del aprendizaje, y el alumno ha pasado de ser un } \\
\text { receptor pasivo de información a un elemento que } \\
\text { participa activamente en su propio aprendizaje. }\end{array}$ \\
\hline
\end{tabular}

Ciencia Latina Revista Científica Multidisciplinar, Ciudad de México, México. 
El Impacto de las Nuevas

Tecnologías en la Boletín de la Red Estatal de

Educación

Superior:

Enfoque

Sociológico
Docencia Universitaria. Vol. 2 de Madrid

El artículo analiza cómo el surgimiento de las TIC en la década de los ochenta ha influido en la creación de un nuevo tipo de sociedad, que recibe el nombre de sociedad informacional, y más concretamente, dentro Tipo básica, de ella, el impacto que han tenido en los cambios nivel educativos y la creciente tendencia hacia el Explicativo. aprendizaje electrónico o e-learning. Los retos principales que las TIC plantean a las universidades es la formación de profesionales para nuevos puestos de trabajo y la formación continua.

En el presente artículo se hace un análisis sobre la implementación de tecnologías de información y comunicación en la educación superior con el objetivo

Tecnologías en la

Educación

Superior: Políticas

2017
N. ${ }^{\circ}$ 3. Universidad Autónoma
Revista Digital

Investigación en de

$\mathrm{y}$
Universitaria. Vol.11 no.1

en

Lima ene./jun. 2017
Torres

implementación.
Melo et al.

Tipo básica,

nivel

descriptivo de aportar conocimiento sobre las tendencias en su apropiación social. Para ello se ha hecho una revisión de documentos de política pública y literatura científica, que contienen las directrices planteadas por organismos internacionales en las cuales se presentan diferentes alternativas para orientar el proceso de integración de las tecnologías en la formación.

La incorporación de las Tecnologías de Información en el aula universitaria requiere una reinvención de la

La Tecnología y la
Educación Superior en época de crisis.
Revista Latinoamericana de Miguel Estudios Educativos
Tipo básica, nivel explicativo.

forma como realizamos la enseñanza y la adaptación
de los docentes y del alumnado en el entendimiento de las formas en que la Tics pueden apoyar en el proceso de enseñanza-aprendizaje. 
Impacto de las TIC en la educación: Revista de Psicología
Educativa. Universidad San Ignacio de Loyola, Lima, Perú.
Tipo básica,

nivel

explicativo.

El presente artículo define los principales retos y muestra un panorama futuro de las TIC y su relación en el ámbito educativo. El inicio está plasmado por la definición, de lo que actualmente se llama sociedad del conocimiento y cómo su evolución, producto de la tecnología, ha abarcado distintas áreas, permitiendo innovar a la educación, estimulando la creación de nuevos conocimientos, de esta manera se describe la importancia de cada agente educativo (docentealumno) y su rol transformador en el proceso de enseñanza-aprendizaje.

En los últimos años la repercusión del uso de las TIC tiende a extenderse rápidamente en el mundo actual, tanto en la economía, como en la educación, recomiendan la introducción de la informática y las telecomunicaciones en la educación, ponen énfasis especial en la necesidad de rediseñar los contenidos de

Las Tecnologías de la información en el cambio de la Educación Superior en el Siglo XXI: Reflexiones para la

Universidad y Sociedad vol.8
no.4 Cienfuegos sep.-dic. 2016.CUBA.
Garcés al. la enseñanza para que permitan ser utilizadas en los Tipo básica, procesos formativos.

nivel

explicativo. Esta posición marca la orientación de las instituciones educativas de todos los niveles educativos, pero incorpora las TIC como recurso estratégico para la transformación educativa y social desde la cual se generen las condiciones necesarias para que todos puedan acceder a la educación y puedan brindar al estudiante la oportunidad de comprometerse con su desarrollo y el de sus semejantes.

Fuente: Elaboración propia (2021).

Ciencia Latina Revista Científica Multidisciplinar, Ciudad de México, México. 
Acorde con el objetivo de revisar diversas investigaciones del período 2018 - 2020 que hacen referencia a la calidad de la educación superior tecnológica en el contexto de Iberoamérica y particularmente en el Perú, se encontró una diversidad de miradas formas de concebir a la educación tecnológica; algunos relacionan la educación con la tecnología desde una perspectiva pedagógica y la empleabilidad, otros enfatizan en el impacto de las NTIC, las innovaciones y desafíos con enfoque sociológico; también destacan algunas políticas públicas y apropiación social en su implementación, entre otras reflexiones para la práctica y su mejora (...) Casi muy poco, se aborda a la educación tecnológica como parte de un sistema educativo que busca otorgar a los estudiantes el desarrollo de habilidades y capacidades técnicas y tecnológicas propias de algún oficio o profesión; sino más bien, enfatiza en su importancia y en la necesidad de aprender o incursionar según las demandas y crisis que la revolución tecnológica genera en cada contexto y está imbricada a la formación general e indistintamente en cada nivel educativo.

Por eso, existen varias divergencias y enfoques al respecto. Acevedo (2018), en su investigación, destaca un enfoque sobre diferentes nodos; primer nodo: la crítica pedagógica al efecto de los medios de comunicación en la educación. segundo nodo: la inclusión en educación con el uso de la tecnología y la implementación de la educación virtual y por último, tercer nodo: la subjetividad y la experiencia de interacción en entornos tecnológicos; a similar conclusión llegaron, Solís y Duarte (2018), quienes indicaron que, la formación tecnológica es altamente especializada y práctica bajo la modalidad dual debido al fomento de competencias, habilidades y destrezas para trabajar en sectores productivos de servicios públicos y privados. La posibilidad de acceder al mercado laboral en corto tiempo es alta; ya que su formación es de 2.5 años. Adquiere una capacidad resolutiva a problemas específicos con soluciones prácticas y rápidas en áreas pertinentes. Se demuestra entonces con los porcentajes que la empleabilidad del estudiante quedaría asegurada para toda esa nueva oferta que es de $60 \%$ de la pronosticada en los años 2018-2019-2020.

En cambio, García et al. (2017), puntualizaron que, a la luz de las nuevas tecnologías han cambiado de manera reveladora las relaciones humanas, las nuevas formas de acceso a las tecnologías de la información y comunicación son ahora el motor de los nuevos paradigmas respecto de cómo se relacionan las personas con nuevas formas de participación social, de control y activismo a través de las redes sociales. Las TIC deben 
servir para apoyar a mejorar las habilidades docentes que deberán permitir que el estudiante sea más responsable de su propio aprendizaje ofreciéndole diversas opciones para que investigue, invente, produzca, colabore, transforme, etc., siempre con el objetivo de formar una nueva generación de ciudadanos innovadores.

Para, Torres (2019) las TIC plantean un paradigma educativo totalmente nuevo que puede traer muchas ventajas, pero hay que tener claro que las claves para el cambio de metodología de la enseñanza radicarán en cómo se utilicen estos nuevos medios. El autor prevé que en los primeros años se tenderá a la convergencia de la enseñanza presencial y virtual y que con los años se hará cada vez menos enseñanza presencial en pro de la virtual. Sin embargo, Melo et al. (2017). las conclusiones apuntan a centrar la atención en los procesos de aprendizaje más que a la inversión en equipamientos, subrayando la importancia de las tecnologías en la educación, en el contexto de la cuarta revolución industrial en la era digital.

Del mismo modo, Miguel (2020), manifiesta que se debe implementar un diseño curricular centrado en el aprendizaje y basado en competencias, una de ellas en el uso de tecnologías de información, como alternativa para lograr los objetivos de formación y pertinencia en relación con los cambios en el entorno. El uso de las tecnologías en la educación superior nos brinda beneficios como el trabajo colaborativo, la gestión del aula no solo es tarea del profesor, las nuevas plataformas tecnológicas integran a todos los actores involucrados para mejorar la experiencia del aprendizaje; se puede monitorear si se cumplen los objetivos de la clase, y los estudiantes pueden compartir sus inquietudes y llevar un seguimiento de sus calificaciones.

Por otro lado, Hernández (2017), puntualizó, el proceso de enseñanza-aprendizaje en el aula, haciendo uso de las TIC, requiere de un conjunto de competencias que el docente debe adquirir con la lógica de sumar una metodología capaz de aprovechar las herramientas tecnológicas, donde la capacitación docente deberá considerarse una de las primeras opciones antes de afrontar nuevos retos educativos. Es un hecho que el aporte de las TIC a la educación y a la sociedad como tal, es la flexibilidad, y la adaptación a un entorno cada vez más cambiante; fue quizás en un inicio el trabajo el principal afectado en este proceso, sin embargo, el trascurrir del tiempo ha evidenciado que la sociedad depende de un enfoque tecnológico que lo ayude a construir y adquirir conocimientos. 
Asimismo, Garcés et al. (2016), refirieron que es necesario reconocer que las demandas actuales de la educación superior a la renovación e innovación curricular que se está gestando a nivel mundial no solo incluye la introducción de las TIC como un medio de enseñanza, sino que estimula un cambio organizativo, metodológico que debe apostar por la integración de estas al proceso de enseñanza-aprendizaje. La búsqueda de soluciones que respondan a este cambio depende de las políticas educativas, de la gestión de cada universidad y del nivel de implicación que tengan los docentes en la utilización óptima de las TIC en las actividades de enseñanza y aprendizaje.

\section{CONCLUSIONES}

La calidad de la educación tecnológica no está muy explicita; por cuanto, existe una heterogeneidad de concepciones y acepciones como proceso formativo y resultados; para algunos, está centrado en el desarrollo de contenidos que deben ser abordados dentro o fuera de un sistema educativo orientados a comprender el uso de las diversas tecnologías en un contexto sociocultural; mediante capacitación, instrucción y desarrollo de habilidades sobre un determinado equipo tecnológico; a fin de entender, primero para qué sirve, cómo funciona, cuál es el propósito para el que fue creado, qué necesita el usuario para su uso adecuado y evitar daños, etc. Para otros, la educación tecnológica forma parte del sistema educativo y está orientado a formar jóvenes calificadas en el manejo y conocimiento de los procesos tecnológicos de los diferentes sectores productivos, mejorar el pensamiento práctico, creativo y crítico, tomar decisiones en la solución de problemas, potenciar la capacidad de organización, la creatividad, la responsabilidad, liderazgo y valores para participar de manera eficiente y eficaz en el desarrollo de la economía nacional.

En ese marco, de una educación tecnológica como parte de un sistema educativo, se deduce que la calidad de la educación superior tecnológica en el Perú y otros países iberoamericanos tiene una baja valoración en el sector productivo y poco prestigio social, debido a su precariedad en el equipamiento y servicio educativo, debido a la escasa articulación con las demandas laborales y las necesidades del desarrollo local, regional y nacional; sumándose otros indicadores educativos o condiciones mínimas de calidad que deben tener las instituciones públicas o privadas que brindan este servicio; propiciando la cabida de los procesos de mejora continua de la calidad para promover el valor de la optimización, el licenciamiento y la acreditación como estrategia para alcanzar la calidad. 
En esa misma línea, la educación universitaria tecnológica posee un compromiso frente a la comunidad, también revelan precariedad y baja calidad; pues, presentan variadas carencias, a nivel de la docencia, los planes de formación de manera transversal, el equipamiento y sobre todo, los nuevos contextos en los que se desempeñarán los futuros profesionales exige actualización e innovación tecnológica en todos sus áreas y facultades; por cuanto, las innovaciones y la formación profesional exigen que los próximos egresados sean creativos, independientes y capaces de decidir responsablemente; y en ese sentido, apoya a la pedagogía que busca la responsabilidad, subjetividad y autonomía del individuo en la formación profesional y en el trabajo.

\section{LISTA DE REFERENCIAS}

Acevedo, S. (2018). (2018). Revisión de la educación y la tecnología desde una mirada pedagógica. Pedagogía y $\quad$ 97-110. https://doi.org/10.17227/pys.num48-7376

Andrade, J. \& Campo-Redondo, M. (2012). Apropiación social de las tecnologías de información: políticas públicas para la participación ciudadana. Quórum Académico, 9(1), 52-68. https://goo.gl/g77ttU

Benavides, F. \& Pedró, F. (2007). Políticas educativas sobre nuevas tecnologías en los países Iberoamericanos. Revista Iberoamericana de Educación, 45, 19-69. http://rieoei.org/rie45a01.htm

Claro, M. (2010). La Incorporación de Tecnologías Digitales en Educación. Modelos de identificación de buenas prácticas. CEPAL. https://goo.gl/5vhQLd

Codina, L. (2009). Ciencia 2.0: Redes sociales y aplicaciones en línea para académicos. Hipertext.net, 7. https://goo.gl/RnhhQa

Gallardo-Echenique, E., Marqués Molías, L., \& Bullen, M. (2015). Students in higher education: Social and academic uses of digital technology. RUSC. Universities and Knowledge Society Journal, 25. https://doi.org/10.7238/rusc.v12i1.2078

Garcés, E., Garcés, E. y Alcívar, O. (2016). Las tecnologías de la información en el cambio de la educación superior en el siglo XXI: reflexiones para la práctica. Revista Universidad y Sociedad, 8(4), 171-177. 
García, M., Reyes, J., \& Godínez, G. (2017). Las Tic en la educación superior, innovaciones y retos. Revista Iberoamericana de las Ciencias Sociales y Humanísticas, 6(12), 299-316.

Hernández, R. (2017). Impacto de las TIC en la educación: Retos y Perspectivas. Propósitos y representaciones, 5(1), 325-347.

Kozma, R. (2008). Comparative analyses of policies for ICT in education. En J. Voogt \& G. Knezek (Eds.). International handbook of information technology in primary and secondary education (pp. 1083-1096). Berlín: Springer. http://doi.org/10.1007/978-0-387-73315-9

López, C. (2011). Políticas públicas y TIC en la educación. CTS: Revista Iberoamericana de Ciencia, Tecnología y Sociedad, 6(18). https://goo.gl/7aJEa5

Lugo, M. (2010). Las políticas TIC en la educación de América Latina: Tendencias y experiencias. Revista Fuentes, 10, 52-68. https://goo.gl/TZot4O

Martí-Noguera, J. \& Martí-Vilar, M. (2015). Responsabilidad social en la educación básica y superior: una perspectiva desde el espacio iberoamericano. Revista de Educação PUC-Campinas, 20(1), 27. https://doi.org/10.24220 / 23180870v20n1a2941

Melo, D., Silva, J., Indacochea, L., \& Núñez, J. (2017). Tecnologías en la Educación Superior: Políticas Públicas y Apropiación Social en su implementación. Revista Digital de Investigación en Docencia Universitaria, 11(1), 193-206.

Miguel, J. (2020). La educación superior en tiempos de pandemia: una visión desde dentro del proceso formativo. Revista Latinoamericana de Estudios Educativos, $50,13-40$.

Ministerio de Educación - MINEDU (2017). Reglamento de la Ley $N^{\circ} 30512$, Ley de Institutos y Escuelas de Educación Superior y de la Carrera Pública de sus Docentes. Gobierno del Perú.

Organization of Economic Co-operation and Development - OCDE. (2016). Política regulatoria y gobernanza: apoyo al crecimiento económico y servicio al interés público. Publicaciones de la OCDE

Rochefort, B. y Richmond, N. (2011). El impacto de las redes sociales en la enseñanza y el aprendizaje. Revista de Universidad y Sociedad del Conocimiento (RUSC). $8(1), 200-216$. 
Calidad de la educación superior...

Salavisa, I. \& Fontes, M. (2012). Redes sociales, innovación y economía del conocimiento. Editorial Routledge.

Solís, M. y Duarte, P. (2018). La educación superior tecnológica y la empleabilidad. Revista Universidad y Sociedad, 10(3), 21-33.

Torres, C. (2019). El Impacto de las Nuevas Tecnologías en la Educación Superior: un Enfoque Sociológico. Boletín de la Red Estatal de Docencia Universitaria, 2(3). 